
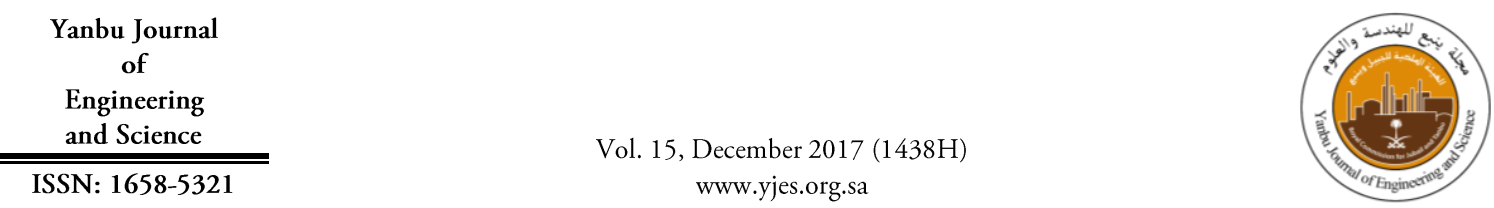

\title{
NUMERICAL EXPERIMENTS ON SHOCK WAVE DIFFRACTION AROUND RAMP SPLITTER USING SMALL AND LARGE SHOCK TUBES
}

\author{
Ahmed M. Bagabir \\ Faculty of Engineering, Jazan University, Jazan, Saudi Arabia \\ E-mail:abagabir@yahoo.com
}

\begin{abstract}
The experimental studies on shock wave diffraction have been done on conventional small-scale experimental shock tube which reveals spatial and temporal limitations. The wave reflected from the walls of the test section interferes with the evolution of the shear layer and its associated vortex. The computational fluid dynamics can simulate flow patterns of large-scale shock tube that are difficult, expensive, or impossible to study using measurements. The present numerical research is dedicated for diffraction of unsteady compressible flow around $172^{\circ}$ ramp splitter using small and four-time larger shock tubes. Both scales are realistic sizes that are likely to be found in the real life applications. Therefore, the objectives of the research are to achieve a better understanding of spiral vortex evaluation, shock-vortex interaction and the associated Kelvin-Helmholtz instability that are not covered by experiment shadowgraphs. The present numerical method is a cell centered finite volume. The second-order AUSM+ scheme is applied to calculate the inviscid fluxes of the unsteady Euler equations. The simulations are performed using quadrilateral mesh. Mesh adaption of five additional levels each refined by a factor of four is applied in regions where density gradients exceed $5 \%$ of the local normalized value. Results are presented for weak and strong shock waves of the Mach numbers 1.31 and 1.59. The numerical results reveal excellent agreement with the corresponding experimental shadowgraphs. It is found that the refracted shock moving inside the vortex is affected by the rotation and centrifugal forces which have not been noted before.
\end{abstract}

Keywords: Shock, Splitter, Compressible, Euler equations, Kelvin-Helmholtz instability.

\section{INTRODUCTION}

Compressible flows around solid surfaces have attracted the interest of many researchers. They are motivated by the need to understand the physics of gas dynamic phenomena which are crucial in the analysis of the performance of aerodynamic flow devices. Flow separation is the detachment of fluid flow from a solid surface. That is due to the formation of an adverse pressure gradient along the surface relative to the flow direction. The flow breaks away from the surface when the pressure gradient is strong enough to bring the shear stress at the surface to zero. Moreover, the curved surface enhances the adverse pressure gradient by the continuous variation of the surface boundary condition [1]. In addition to the geometry of the solid surface, it is found that the resulting flow depends on the intensity

Received: 20 Dec 2017, Accepted: 31 Dec 2017 
of the shock wave [2-4]. As shown in Figure 1(a), the diffraction of incident shock IS, of Mach numbers less than 1.35 around splitter produces diffracted, DS, and reflected (RS) shocks in the perturbed subsonic region. The separated shear layer, SL, rolls up into a spiral vortex, $\mathrm{V}$, which broadens with flow evolution at later times. Contact surface, CS, moves forward behind the incident shock, Figure 1. As the shock intensity increases, the evolving flow becomes more complex and produces extra feature such as triangleshaped lambda shock, TL, Figure 1(b) [2-8]. Moreover, it is found that the shock-shock and shock-vortex interactions generate vortex embedded shock and secondary shock. The post-shock flow remains subsonic for an incident Mach number less than 2.068. For supersonic post-shock flow, Prandtl-Meyer reflected expansion waves are formed [8]. It is worth mentioning that no additional flow features appear for Mach numbers greater than 3.0. However, the flow features become clearly evident as the shock strength increases [2]. On the other hand, the previous experimental $[4,9,10]$ and numerical $[1,5,6]$ studies have shown Kelvin-Helmholtz instability. It is small vortices forming on the shear layer and rolling-up with the main vortex. Experimental studies on shock wave diffraction have spatial and temporal limitations due to the small size of the shock tube. These limitations result from wave reflection from the walls of the test section moving backward to the perturbed region. The reflected shocks interfere with the evolution of the shear layer and its associated vortices. The experimental studies on shock wave diffraction in shock tubes have been done on conventional small-scale experimental facilities. Nevertheless, recently, Skews et al. [7] repeated the previously published experimental work of Skews [2] on a large-scale shock tube which is five times larger than the conventional one. They tested shock wave diffraction over different angles sharp corners for incident Mach numbers of 1.3-1.5 without the interference of test-section walls. It was reported significant changes in flow evolution as the spatial and temporal scale of the experiment increases [7]. Their breakthrough conclusion was that the flow behind the incident shock is not self-similar as shown in the previous small-scale shock tube experiments. The experimental shadowgraphs showed qualitatively the shock diffraction phenomena occurring during the interaction, but could not reveal details about various shocks, diffractions, and reflections. On contrary, the computational fluid dynamics (CFD) gives an insight into flow patterns that are difficult, expensive or impossible to study using measurements. CFD can simulate virtually any actual flow domain and realistic operating conditions. It is not restricted to laboratory-scale model. Moreover, now a day, CFD predicts results for all desired quantities with high resolution in space and time. The present numerical research is dedicated for diffraction of unsteady compressible flow around $172^{\circ}$ ramp splitter for small and large shock tubes. Both scales are more realistic sizes that are likely to be found in the real life applications. The numerical experiments are conducted with and without the interference of the reflected waves from boundary walls by using small and four-time larger-scale shock tubes, respectively. The large-scale shock tube model allows the flow evolution at longer times without interaction with the reflected waves from the shock tube. The model of small-scale shock tube is similar to the experiments of Gnani et al. [4]. The 
present simulation is applied to interpret and to gain a more solid understanding of the ongoing processes of the complicated flow fields that are not covered by experiment shadowgraphs. Moreover, the effects of intensity of shock wave are investigated by considering weak and strong incident shock of Mach numbers of 1.31 and 1.59 .

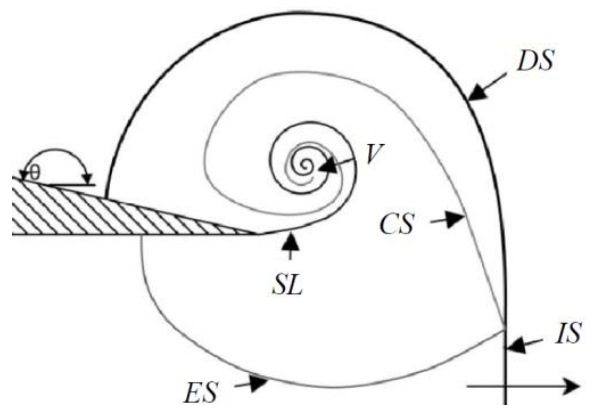

Fig. 1.(a). $1<M \leqslant 1.35$ Flow structures for shock wave diffracting around ramp splitter.

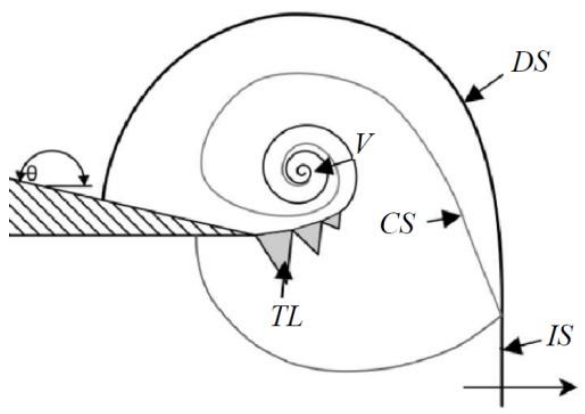

Fig. 1(b). $1.35<M \leqslant 1.8$ Flow structures for shock wave diffracting around ramp splitter.

\section{PROBLEM DEFINITION}

Similar to Gnani et al. [4], an incident shock wave moves around ramp splitter of angle $\theta=172^{\circ}$, Figure 2 . The splitter has height, $h$, and length of $18 \mathrm{~h}$. The large shock tube has height of $17.6 \mathrm{~h}$ which is four times larger than the experimental shock tube as shown in Figure 2. The large-scale model is used to prevent the upper-wall reflected shocks from the intervention of the real flow development. The upper and lower boundaries are rigid non-penetrable walls. Adiabatic conditions are used for solid walls. On the other hand, supersonic flow conditions are set at the inlet section and non-reflecting conditions are set for outlet section. A planar shock wave of Mach number, $M$, moves from left to right into an ambient air at rest. Initially, the pressure, temperature, and velocity, ahead of the shock wave are $1 \mathrm{~atm}, 300 \mathrm{~K}$, and $0 \mathrm{~m} / \mathrm{s}$, respectively. The conditions behind the shock front are related by the RankineHugoniot relations. A perfect gas is assumed and the ratio of specific heats is considered to be 1.4 and air gas constant is $287 J / \mathrm{kgK}$. The present study investigates the flow field for two incident shock waves of the Mach number, $M$, of 1.31 and 1.59 . The Mach number is based on the height of the splitter. The shock wave interactions at these Mach numbers produce distinguished evolving flows. Moreover, they are likely to be found in the real life flight speed of aerodynamic and acoustic devices.

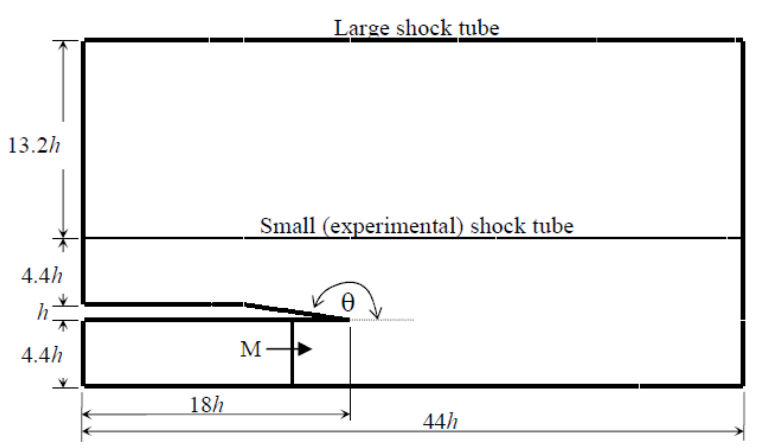

Fig. 2. Geometry and flow conditions for shock wave diffraction around splitter.

\section{METHODOLOGY}

The numerical investigation has been performed using the commercial code ANSYS Fluent Release 13. It is a cellcentered finite volume method. The Euler equations for two-dimensional unsteady compressible flow are employed as the mathematical model. The CFD methodology is to approximate (discretise) the governing partial differential equations into a set of algebraic system, which can be 
solved numerically. One of the challenges in the numerical simulation of compressible flows is to eliminate the numerical uncertainties due to cell size and scheme resolution which can be responsible for suppressing the flow-field details. However, the accuracy of the results is limited by the available computing facilities. Therefore, the present investigation has been performed using high-resolution scheme in association with the optimized cell size. The densitybased solver is employed where the continuity equation is used to obtain the density field while the pressure field is determined from the ideal-gas law equation of state. The Gauss-Seidel linear equation solver is employed in conjunction with an algebraic multi-grid method. The computations are carried out using the Advection Upstream Splitting Method $(\mathrm{ASUM}+)$ [11]. It computes a cell interface Mach number based on the characteristic speeds from the neighboring cells. The interface Mach number is then used to determine the upwind extrapolation for the convection part of the inviscid fluxes at the cell center. The AUSM+ scheme requires the initial values of flow variables at the faces of cells. A second-order least-squares cell-based scheme has been selected for the flow spatial discretization where the gradients are estimated within each cell, and then the face values are obtained. The gradients are subjected to a slope limiting procedure to prevent the creation of local extremes at the boundaries; otherwise, the solutions obtained will exhibit oscillations at shocks. The non-differentiable limiter which maintains stability in the upwind discretization schemes is selected [12]. It uses a minimum modulus function to limit over and undershoots when reconstructing values at the cell faces. The limiter is adequate for a first-order solution across the various discontinuities. The transient terms are discretized by explicit time stepping based on the Courant-Friedrichs-Lewy (CFL) number which is set to one. The simulations are performed using quadrilateral mesh. The base level mesh has 13,919 and 32,345 cells for the small and large shock-tube cases, respectively, Figure 2. Mesh adaption of five additional levels each refined by a factor of four is applied in regions where density gradients in all directions exceed $5 \%$ of the local normalized value. It is found that base level cell size of $1 m m \times 1 m m$ with adaption technique, which provides high resolution of the discontinuities, is adequately capture shock wave features. The post processing of the simulation results is performed by the Tecplot 360 software. The numerical schlieren images are obtained by superimposing a gray-scale color map of the absolute value of the density gradients.

\section{RESULTS AND DISCUSSION}

\subsection{LARGE-SCALE CASES}

Firstly, the results of the large shock tube are presented since the spiral vortical structure is free to develop with no intervention with any reflected shocks from the shock tube walls. A set of numerical schlieren images of a shock wave of $\mathrm{M}=1.31$ diffraction around $172^{\circ}$ ramp splitter is presented in Figure 3. For all presented results, the zero time is considered when the incident shock arriving the sharp corner of the splitter. Figures 2(a) and 3 shows the general flow features of the planar incident shock, IS, diffraction around ramp splitter. The curved diffracted shock, $D S$, which is centered at the corner, propagates upwards. The curved reflected expansion wave, $E S$, travels downwards into the post-shock region. The incident wave, 
which is not affected by the presence of the splitter, meets the curved diffracted shock and expansion wave. The shear layer, $S L$, separates the stationary flow in the expansion region from the conditions behind the shock front. It rolls up into a vortical structure, $\mathrm{V}$, downstream of the splitter, Figures 2 and 3. The contact surface, $C S$, moves forward behind the incident shock. Its upper end is terminated and dragged around the spiral vortex. Moreover, an interesting feature that the KelvinHelmholtz, KH, instability is developed on the shear layer surrounding the vortex, Figure 3. It is associated with the roll-up of small vortices along the shear layer, SL. This can be attributed to the perturbation of the shear layer by the contact surface, $C S$, interaction which makes shear layer unstable forming a series of small vortices rolling-up with the main vortex. Similar instability was previously observed experimentally $[4,7]$ and numerically [5]. As time progresses, the last two frames of Figure 3 illustrate that the shear layer expands and the spiral vortical structure broadens with flow evolution. Higher Mach number of 1.59 reveals discernible distinct flow behavior as depicted in Figures 2 and 4. It is obvious that the vortical structure is bigger than the $\mathrm{M}=$ 1.31 case. In addition to the flow feature of $\mathrm{M}=1.31$, the stronger shock wave diffraction reveals lambda shocks, $T L$, from the early stages of the shock wave diffraction. The lambda shocks, $T L$, comprise a series of triangle-shaped train of expansion waves around the downstream edge of the shear layer, $S L$, starting from the sharp corner of the splitter, Figure 4. Moreover, $\mathrm{M}=1.59$ case reveals right and left vortex shocks, RVS and LVS, inside the spiral vortical structure. The RVS is developed early $(\mathrm{t}=90 \mu \mathrm{s})$ connecting the vortex core and the downstream edge of the shear layer. While, the left vortex shock, LVS, is generated at later time $(\mathrm{t}=253 \mu \mathrm{s})$ connecting the vortex core and the upstream edge of the shear layer. In contrast to the case of shock wave over a sharp corner $[7,8]$ where only one vortex shock is developed connecting the vortex core and the sidewall of the sharp corner. The vortex shocks, RVS and LVS, are deformed and stretched by the rotation effect and centrifugal force of the rotating vortical structure. Consequently, the RVS generates many series of concave pressure waves, CW, which transmit from the spiralvortex as shown in the magnified numerical schlieren image at $\mathrm{t}=270 \mu$ s of Figure 4. At later the time, the $\mathrm{KH}$ vortices still visible but weaker than the ones of $\mathrm{M}=$ 1.31 shock wave.

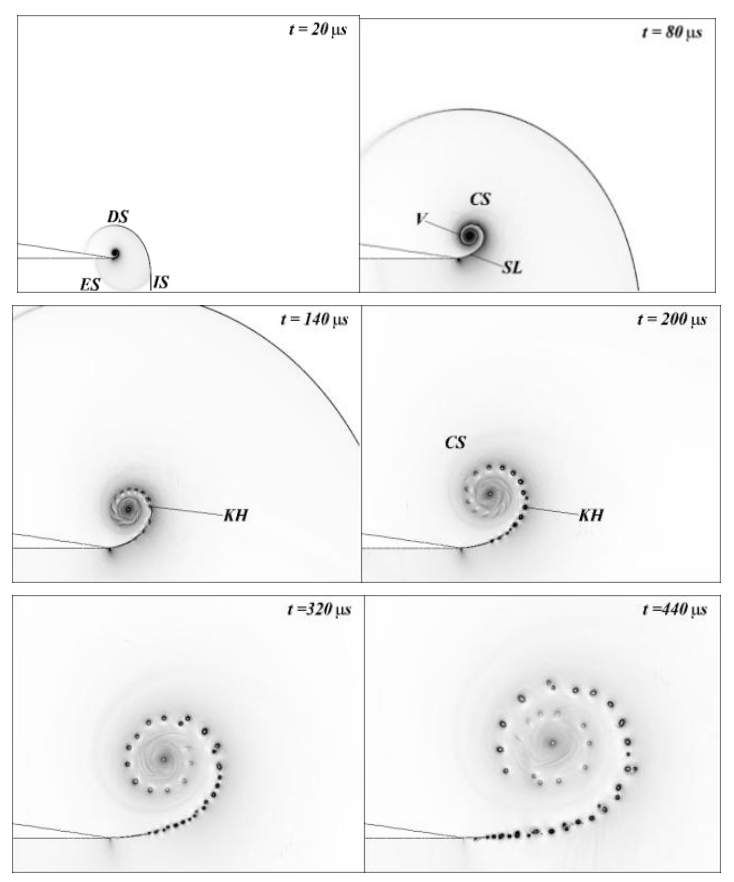

Fig. 3. Numerical schlieren images of shock wave of $M=1.31$ for large-scale case. 


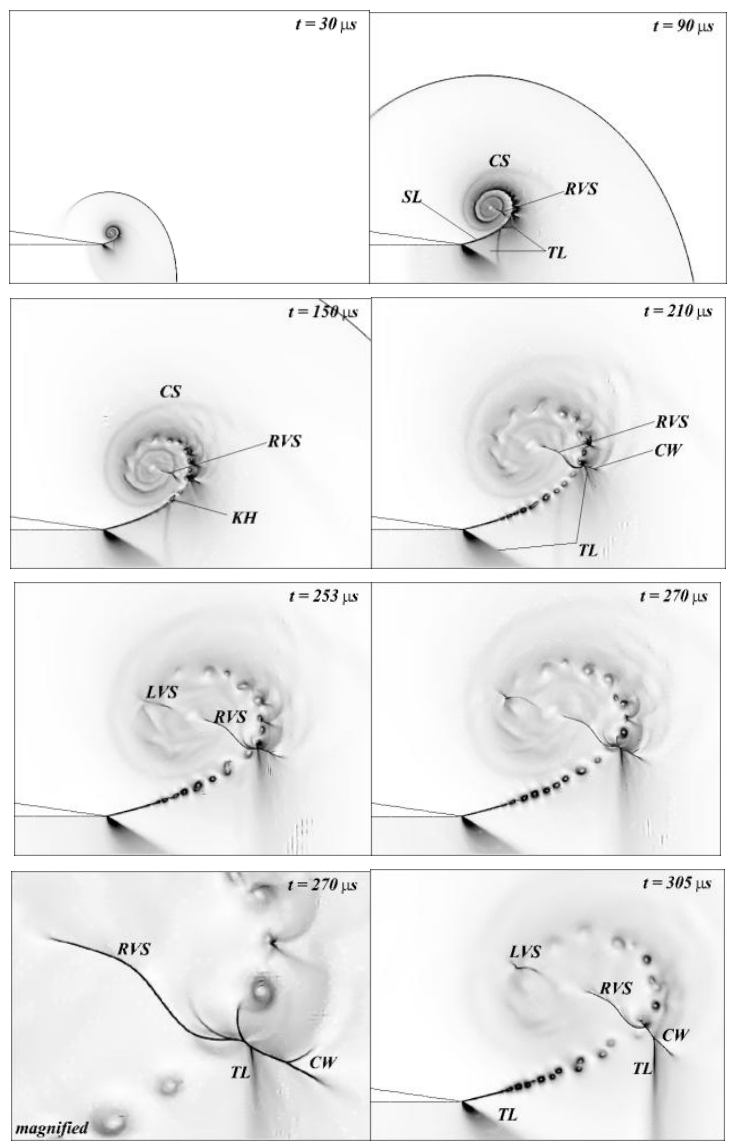

Fig. 4: Numerical schlieren images of shock wave of $M=1.59$ for large-scale case.

\subsection{SMALL-SCALE CASES}

Figures 5 and 6 show sequence of numerical schlieren images of the shock wave of $\mathrm{M}=$ 1.31 and $M=1.59$, respectively, diffraction around $172^{\circ}$ ramp splitter using small shock tube. Comparing with the corresponding set of experimental shadowgraphs [4], the numerical simulation reveals excellent results. It is found that the formation of shock features is well captured in terms of shape, size, and position with time. The generated flow fields are more complicated compared with the large-scale cases shown in Figures 5 and 6. That is due to the multiple interactions of the reflected shocks with the spiral vortical structure. Hence, Figure 7 is dedicated for shading lights on the complex shock-vortex interactions since the experimental shadowgraphs have not revealed the detailed illustration.

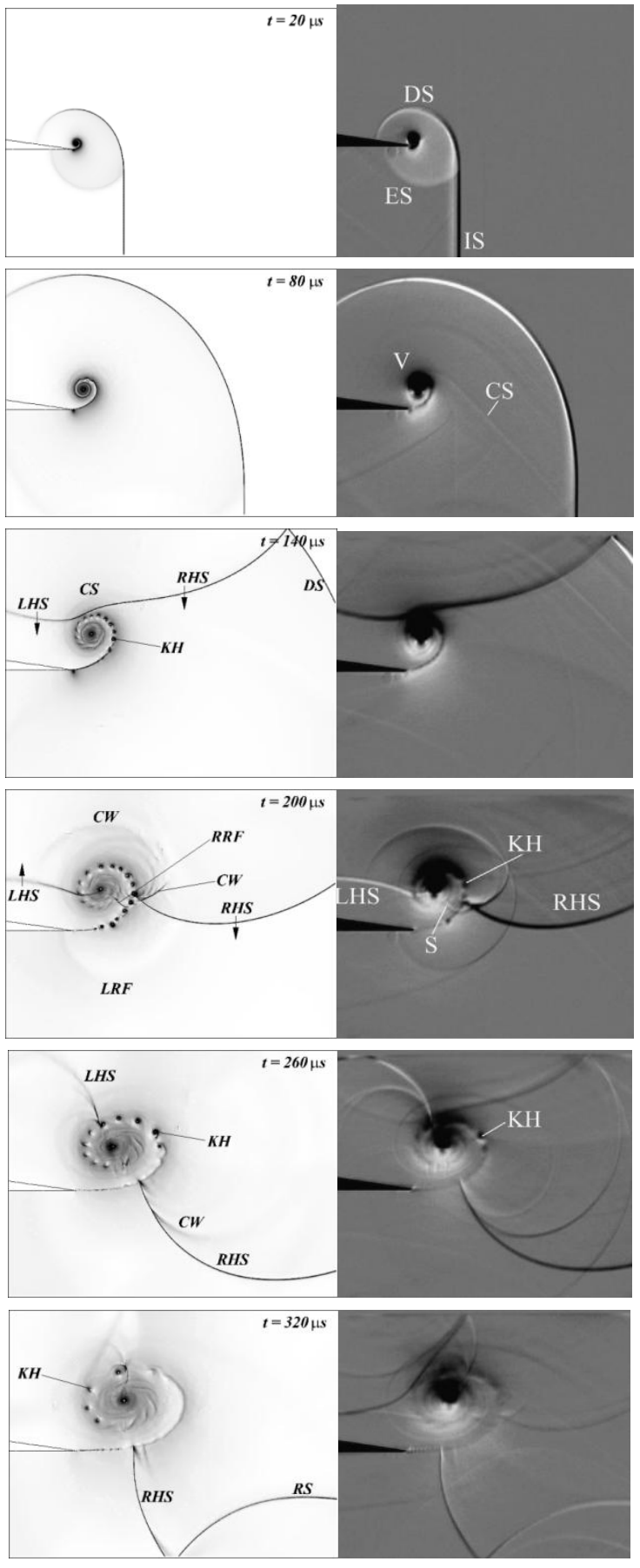

Fig. 5. Numerical schlieren images and experimental shadowgraphs [4] of shock wave of $M=1.31$ for small-scale case.

Also, Figure 7 represents a direct comparison of the vortex evolution for weak and strong shock waves. The effect of the small shock tube starts when the diffracted shock, DS, reflects from the upper wall surface of the shock tube, Figures 5 and 6. The curved reflected shock, RS, is distorted as approaching the vortex because it is moving 
in different sound speed regions [13]. The left segment of the reflected shock, moving in the upstream above the splitter, is faster than the right segment due to the higher sound speed, Figure $5(\mathrm{t}=140 \mu \mathrm{s})$. The reflected shock interaction with the vortex is reminiscent of shock wave impinges on a light bubble $[13,14]$. The interaction produces left-hand shock, LHS, and righthand shock, RHS, and refracted shock, RF, moving inside the main vortex, see the first row of Figure 7 . The RF is affected by the presence of the rotation and centrifugal forces of the spinning vortical structure. As a consequence, the RF initially is separated into two segments. The left one, LRF, follows the vortex rotation and the right segment, RRF, opposes the rotation. The RRF was interpreted by Gnani et al. [4] as stem shock, S. Initially, each segment of the refracted shock is intact with its reflected shock. However, the longitudinal diffused LRF shock transmitted earlier from the vortex. The RRF remains inside the vortex. As time progresses, it breaks into concave shock segments due to rotation effect. They transmit out of the vortex affected by the presence of centrifugal force. The transmitted shocks generate concave pressure waves, CW, surrounding the spiral vortical structure which is more pronounced in the experimental shadowgraphs, Figure 7. The evolving flow of $M=1.59$ case becomes more complex due to the formation of the vortex shocks, RVS and LVS, and multiple interactions. The RVS which is created earlier is taken over by the RRF, Figure 7 ( $t$ $=180 \mu \mathrm{s})$. However, LVS is stretched out after the interaction with the reflected LHS, Figure $6(\mathrm{t}=210-270 \mu \mathrm{s})$. The KelvinHelmholtz, $\mathrm{KH}$, instability is also developed on the shear layer surrounding the vortex, Figures 5 and 6.

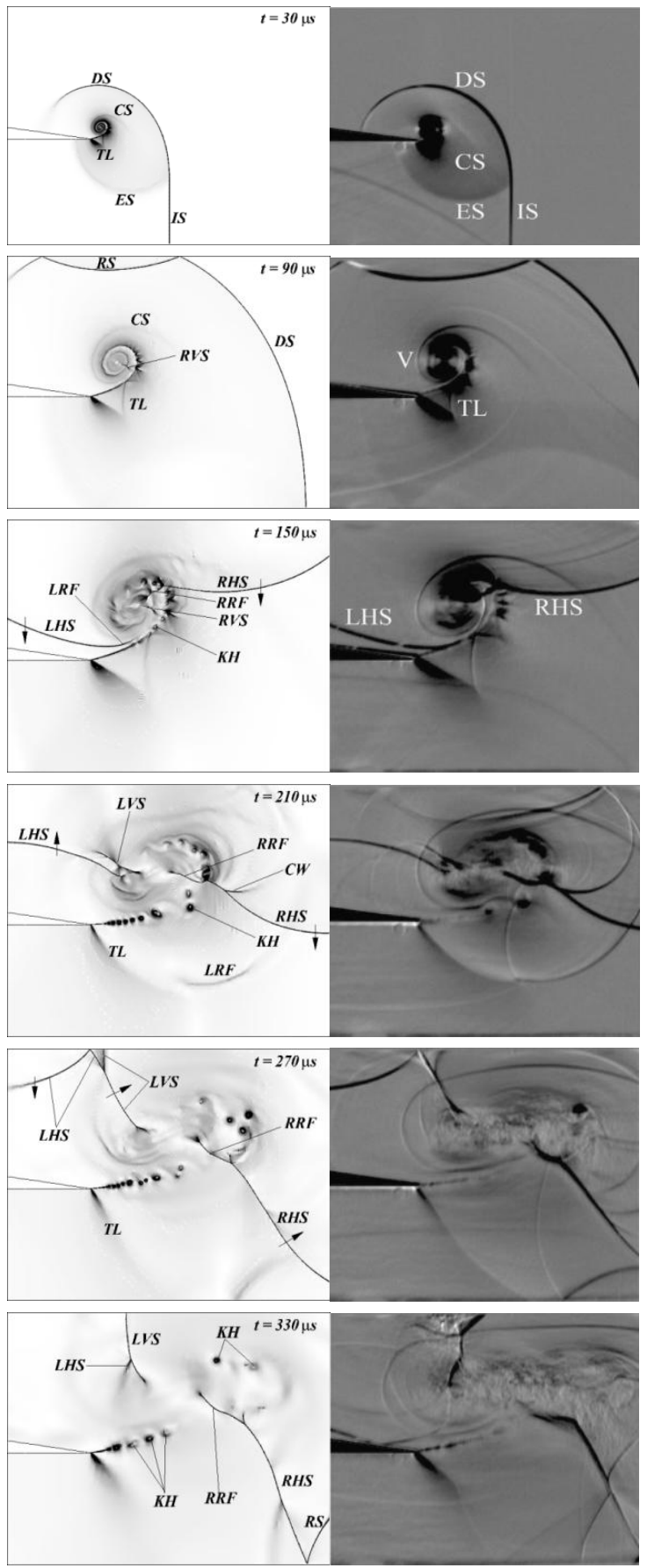

Fig. 6. Numerical schlieren images and experimental shadowgraphs [4] of shock wave of $M=1.59$ for small-scale case.

The $\mathrm{KH}$ instability is more pronounced in the numerical results. Gnani et al. [4] interpreted the formation of the $\mathrm{KH}$ instability due to the interaction of the vortex with the reflected shock, RS, from the upper surface of the shock tube. However, the large-scale cases illustrate that the $\mathrm{KH}$ instability developed even without the 
interaction of the vortex with the reflected shock. As time progresses, the last two frames of Figures 5 and 6 illustrate the enlargement and distortion of the circular vortical structure while moving downstream. The vorticity of the small-scale cases evolves differently from large-scale cases showing an elongated structure which is associated with the multiple shock interaction. Moreover, the higher upstream velocity of a higher Mach number enhances the rate of dissipation of the vortices. It elongates the shear layer and formulates the elliptical vortex shape.

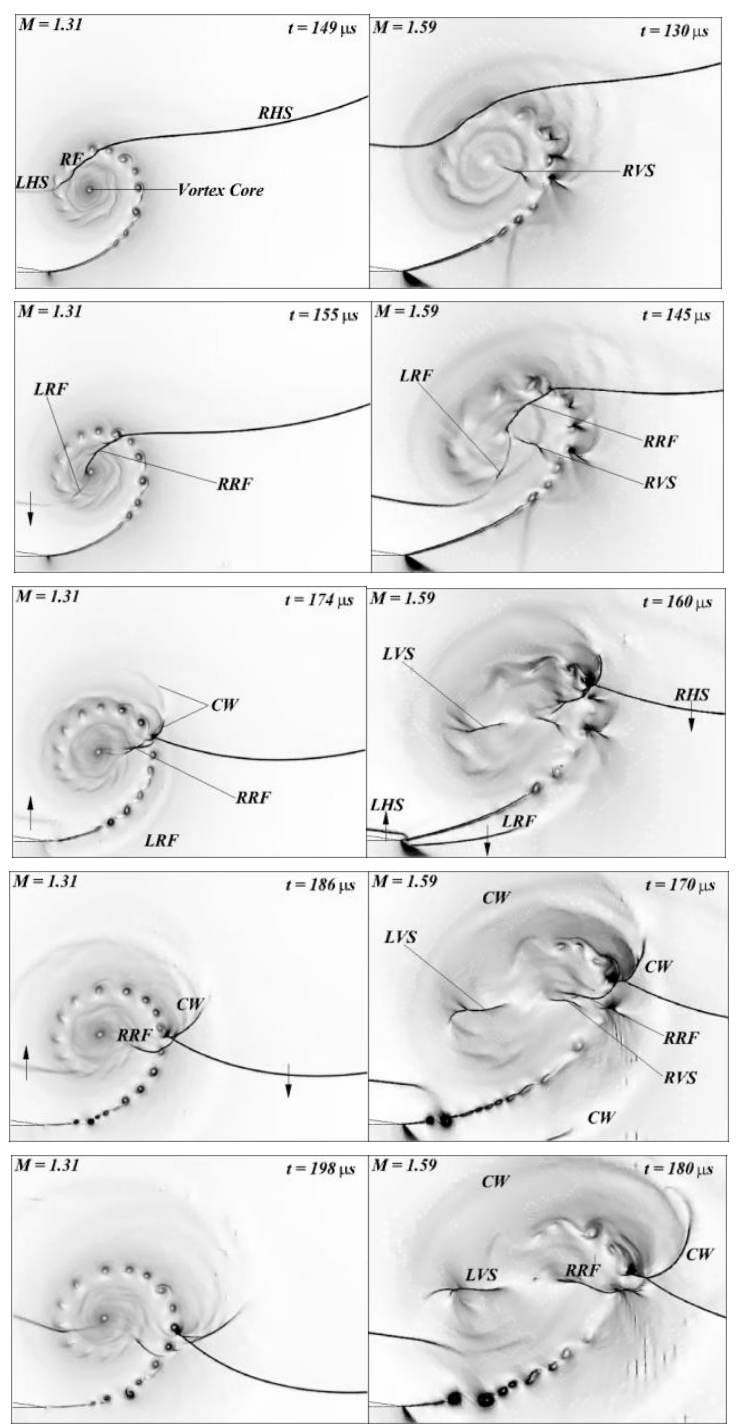

Fig. 7. Numerical schlieren images showing shock-vortex interactions for $M=1.31$ (left) and $M=1.59$ (right) for small-scale case.

\subsection{QUANTITATIVE COMPARISON}

The path of the vortex core of weak and strong shock waves diffraction around $172^{\circ}$ ramp splitter is shown in Figure 8 for smallscale and large-scale cases. The original point $(0,0)$ refers to the location of the splitter sharp corner. The vortex core is the centre of the main vortex which is visible in Figure 7. Its position is digitized from a sequence of numerical schlieren images. The distance is normalized by the height of the splitter, $h$. The present numerical results show quantitatively excellent agreement with the experimental result [4], Figure 8. Moreover, the variations of instant horizontal and vertical velocities of the vortex core with the time for all cases are shown in Figure 9. The velocity is normalized by the corresponding shock velocity. It is obvious that the shockshock and shock-vortex interactions affected the acceleration and deceleration of the vortex after a certain period of time due the fluctuating flow field in the perturbed region, Figure 9. Initially, the vortex of the weak and strong shock waves diffracted on the large shock tube moves in a straight line with almost fixed inclination, Figure 8. As time progresses, the vortex core decreases its slope significantly. The angle that the vortex core makes with respect to the horizontal changes from $63^{\circ}$ to $47^{\circ}$ at $\mathrm{t}=145 \mu \mathrm{s}$ and from $51^{\circ}$ to $36^{\circ}$ at $t=208 \mu$ s for the weak and strong shock waves, respectively, Figure 8. Therefore, the vortex core of the $M=$ 1.31 case moves in about $12^{\circ}$ higher inclination than $M=1.59$ case. The vortex of the large shock tube travels faster. Although, it is difficult for the small shock tube to follow the path of the vortex core particularly for strong shock case; however, the shock reflected from the upper wall compresses the vortex and changes its path, Figure 8. It is found that the vortex moves at 
pathway of about $22^{\circ}$ and $4^{\circ}$ for weak and strong shock cases, respectively. Therefore, the reflected shock squeezes considerably the vortex downwards $25^{\circ}$ and $32^{\circ}$ for weak and strong shock waves, respectively. This is clear from the vertical-direction deceleration of the vortex core, Figure 9.
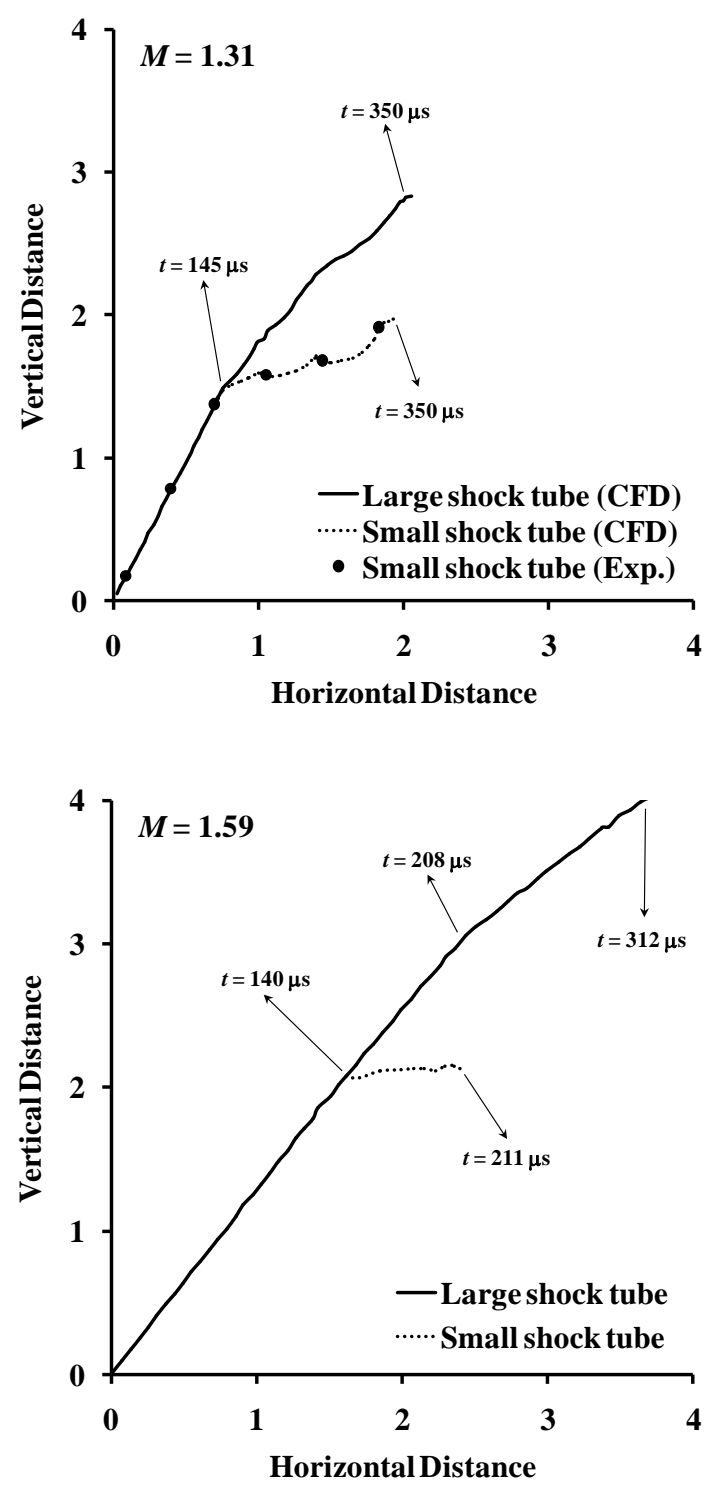

Fig. 8: Location of the vortex core of small- and large-scale cases for $M=1.31$ (top) and $M=1.59$ (bottom).
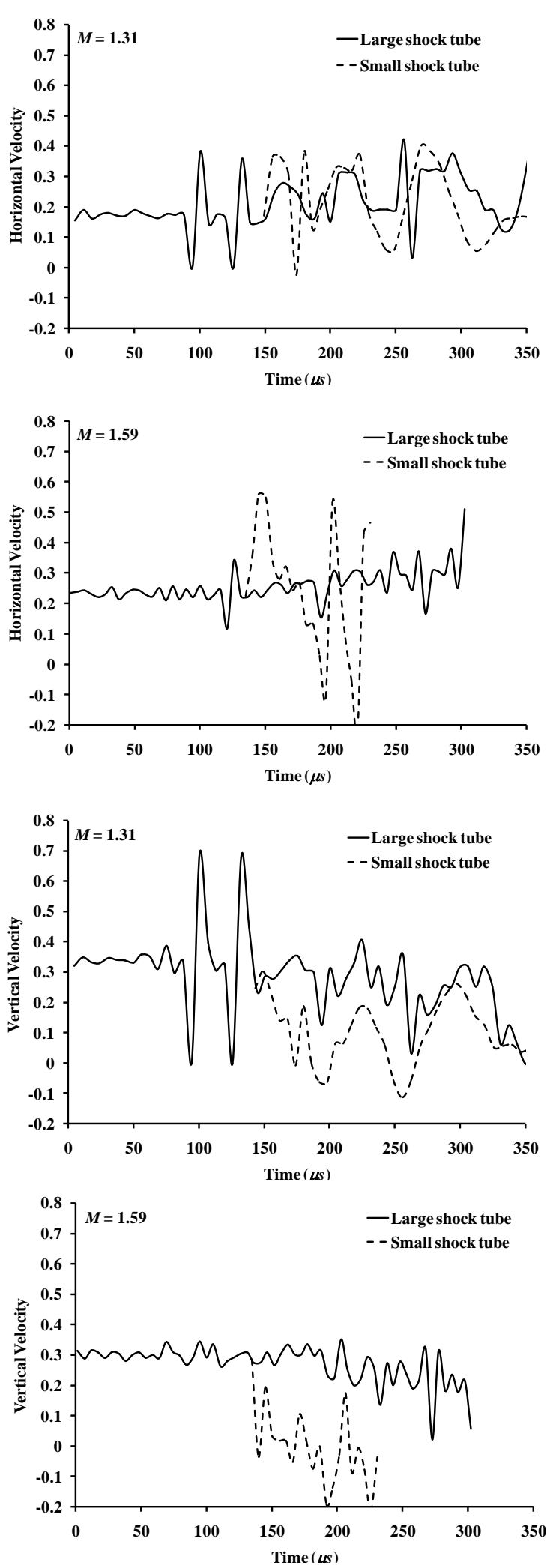

Fig. 9: Variations of horizontal and vertical velocities of the vortex core for different cases. 


\section{CONCLUSION}

The main findings of the present numerical investigation are summarized as follows: Numerical experiments have performed for diffraction of unsteady compressible flow around $172^{\circ}$ ramp splitter in small and large shock tubes for weak and strong shock waves of the Mach number 1.31 and 1.59, respectively. The present numerical research simulates compressible flow on large-scale shock tube which is difficult to be done experimentally. The large-scale cases facilitate the understanding of the complicated shock interaction in the smallscale shock tube. The numerical simulations interpret and gain a solid understanding of the ongoing processes of the complicated flow fields of shock-shock and shock-vortex interactions that are not covered by experiment shadowgraphs. The numerical results reveal excellent qualitative and quantitative agreement with the corresponding experimental results. All cases studied exhibit the Kelvin-Helmholtz instability which is attributed to the perturbation of the shear layer by the contact surface. The strong shock wave of $M$ $=1.59$ diffraction reveals two embedded vortex shocks, RVS and LVS. The vortex and refracted shocks are greatly influenced by the rotation effect and centrifugal force of the spinning vortical structure. The rotation force breakdowns and elongates the shocks inside the vortex; while the centrifugal force transmits the shocks out of the spiral vortical structure. For small-scale cases, the refracted shock, $R F$, breakdowns in two shock segments, $L R F$ and $R R F$. The right vortex shock, $R V S$, is merged with the right refracted shock, $R R F$. The $L R F$ and concave shock segments generated from $R R F$ transmit out of the vortical structure. The transmitted shock waves generate concave pressure waves, $C W$, surrounding the vortex. The flow with the higher Mach number causes the vortical structure to be more inclined with respect to the horizontal. The upper-wall reflected shock significantly compresses the vortical structure downwards.

\section{ACKNOWLEDGEMENT}

The DAAD fund of visiting scholar (50015559) at the Magdeburg University, Germany, is appreciated.

\section{REFERENCES}

[1] Law, C., Muritala, A. O. and Skews B. W., Unsteady flow with separation behind a shock wave diffracting over curved walls, Shock Waves, 24 (3), 283-294, 2014.

[2] Skews, B.W., The perturbed region behind a diffracting shock wave, J. Fluid Mech., 29, 705-719, 1967.

[3] Bagabir, A., Abdulrahman A. and Balabel, A., Shock-wave diffraction over single and double wedges, Emirates Journal of Engineering, 12 (2), 53-66, 2007.

[4] Gnani, F., Lo, K.H., Zare-Behtash, H. and Kontis, K., Experimental investigation on shock wave diffraction over sharp and curved splitters, Acta Astronautica, 99, 143-152, 2014.

[5] Takayama, K. and Inoue, O., Shock wave diffraction over a 90 degree sharp corner, Shock Waves, 1, 301-312, 1991.

[6] Sun, M. and Takayama, K., A note on numerical simulation of vortical structures in shock diffraction, Shock Waves, 13, 25-32, 2003.

[7] Skews, B., Law, C., Muritala, A., and Bode, S., Shear layer behavior resulting from shock wave diffraction, Exp. Fluids, 52, 417-424, 2012.

[8] Bagabir, A., Comparison of compression and blast waves diffraction over $90^{\circ}$ sharp corner, Aljouf University Science and Engineering Journal, 3 (2), 10-20, 2016.

[9] Quinn, M.K. and Kontis, K., A combined study on shock diffraction, 5th Symposium on Integrating CFD and Experiments in Aerodynamics, Japan, 2012. 
[10] Gnani, F., Lo, K.H., Zare-Behtash, H. and Kontis, K., Shock wave diffraction in the presence of a supersonic co-flow jet, Shock Waves, 26, 253-262, 2016.

[11] Liou, M. S., A sequel to AUSM: AUSM+, J. Comput. Phys., 129, 364-382, 1996.

[12] Barth, T. J. and Jespersen, D., The design and application of upwind schemes on unstructured meshes, Technical Report AIAA-89-0366, AIAA 27th Aerospace Sciences Meeting, Reno, Nevada, 1989.
[13] Bagabir, A. and Drikakis, D., Mach number effects on shock-bubble interaction, Shock Waves, 11, 209-218, 2001.

[14] Bagabir, A. and Balabel, A., A level set method for tracking interfaces in unsteady compressible flow. Emirates Journal of Engineering, 15 (1), 13-23, 2010. 


\title{
التحليل العددي لمحاكات إنتشار موجة تصادمية حول سطح مائل داخل أنبوب صغير و كبير
}

\author{
أحمد محمد باجابر
}

كلية الهندسة ، جامعة جاز ان، جاز ان، المملكة العربية السعودية.

الملخص:

وجد أن التجارب العملية لإنتشار موجة تصادمية داخل أنبوب صغير محددة بمساحة ضيقة ولا يمكن إجراء تجارب لفترة زمنية طويلة لان الموجة المرتده من الجدران الخارجية تعيق تطور الموجات الأصلية الناتجة عن الانتشار، لذا في هذه الدراسة تم عمل نموذج التحليل العددي لمحاكات انتشار وتصادم موجتين ضعيفة (M=1.59) و (M=1.31) حول سطح صلب مائل بز اوية 172 درجة داخل أنبوب كبير. أجريت الدر اسة أيضا لحالة الأنبوب الصغير للمقارنة. النموذج المستخدم يعتمد على طريقة العناصر الحجمية المحدة و الممثلة فى نظام أحداثي مطابق للجسم ثنائي الأبعاد. حيث تم حل معادلات أويلر للغاز ات المضغوطة بو اسطة الحلول الضمنية للزمن. وقد أخذ بعين الأعنبار تحويل المعادلات التفاضلية الى منظومة من المعادلات الجبرية التى يسهل حلها. نم التأكد من دقة نموذج التحليل العددي بالمقارنة مع نتائج عملية سابقة. النتائج وضحت أن الموجات التصادميه تودي الى أنظمه مختلفة معقدة من الموجات نسبةً لتصادمها مع بعضها البعض. 